\title{
Hadroproduction in high energy collisions
}

\author{
Alexander Bylinkin* \\ Moscow Institute of Physics and Technology (MIPT), Moscow, Russia \\ E-mail: alexander.bylinkin@gmail.com
}

Transverse momentum spectra, $d^{2} \sigma /\left(d \eta d p_{T}^{2}\right)$, of charged hadron production in $p p$-collisions are considered in terms of a recently introduced two component model. The shapes of the particle distributions vary as a function of c.m.s. energy in the collision and the measured pseudorapidity interval. In order to extract predictions on the double-differential cross-sections $d^{2} \sigma /\left(d \eta d p_{T}^{2}\right)$ of hadron production for future LHC-measurements the different sets of available experimental data have been used in this study. Finally, possible interpretation of the observed phenomena is presented.

The European Physical Society Conference on High Energy Physics

5-12 July

Venice, Italy

${ }^{*}$ Speaker. 


\section{Introduction}

There exists a large body of experimental data on hadron production in high energy protonproton, photon-proton, photon-photon and heavy-ion collisions. The spectra of hadrons produced in these collisions are characterized by an exponential behavior as a function of transverse momenta, $p_{T}$, for the bulk of produced hadrons, which populate the low $-p_{T}$ part of the spectra. This behavior resembles the Boltzmann-like spectrum in classical thermodynamics. The exponential shape of the spectra changes to a power law for high $-p_{T}$ hadrons. This change is traditionally interpreted as an onset of the perturbative QCD regime of hadron production. These features of the spectra shape are found to be universal for any type of colliding particles. Therefore, it is tempting to find one universal smooth functional form, which will describe the spectra of produced hadrons in the whole available $p_{T}$ range for various experimental setup. The parameters of such universal functional form are expected to vary for different collision energies, types of colliding particles and types of produced hadrons. Studies of their variations provide a unique information on the hadron production dynamics.

Recently, such phenomenological model considering two sources of hadroproduction has been introduced [1]. It was suggested to parametrize charged particle spectra by a sum of an exponential (Boltzmann-like) and a power-law $p_{T}$ distributions:

$$
\frac{d^{2} \sigma}{d \eta d p_{T}^{2}}=A_{e} \exp \left(-E_{T k i n} / T_{e}\right)+\frac{A}{\left(1+\frac{p_{T}^{2}}{T^{2} \cdot N}\right)^{N}},
$$

where $E_{T k i n}=\sqrt{p_{T}^{2}+M^{2}}-M$ with $M$ equal to the produced hadron mass. $A_{e}, A, T_{e}, T, N$ are free parameters to be determined by a fit to the data. The detailed arguments for this particular choice are given in [1]. The exponential term in this model is associated with thermalized production of hadrons by valence quarks and a quark-gluon cloud coupled to them. The power-law term is related to the mini-jet fragmentation of the virtual partons (pomerons in $\mathrm{pQCD}$ ) exchanged between two colliding partonic systems.

A typical charged particle spectrum as a function of transverse momentum measured by the Alice Collaboration [7] and fitted with this function (1.1) is shown in figure 1. As one can see, the exponential term dominates the particle spectrum at low $p_{T}$ values.

Separating "soft" and "hard" contributions with this model allowed to calculate the predictions on the mean $\left\langle p_{T}\right\rangle$ values as a function of multiplicity in a collision [2] and pseudorapidity distributions of charged particles. However, the major interest of many studies in QCD is the transverse momentum spectrum itself. Therefore, in this article it is discussed how its shape varies in different experiments under various conditions. In [1] it was shown that the parameters of the fit (1.1) show a strong dependence on the collision energy. Unfortunately, due to the fact that different collaborations measure charged particle production in their own phase space and under various experimental configurations, the dependences observed in [1] were smeared and did not allow to make strong predictions for further measurements. Thus, an approach to correct the measurements in order to allow an accurate combination of different experimental data is proposed here. 


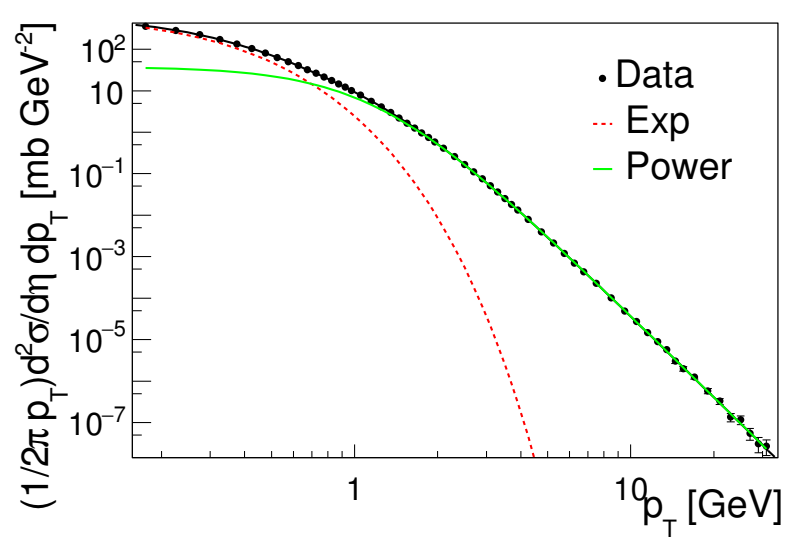

Figure 1: Charge particle differential cross section [7]: the red (dashed) line shows the exponential term and the green (solid) line shows the power law term.

\section{Parameter variations}

In [3] it was shown that two sources of hadroproduction described above contribute to different pseudorapidity regions: while the power-law term of (1.1) prevails in the central rapidity region $(|\eta| \lesssim O(1))$, the exponential term dominates at high values of $\eta$. The idea to study parameter variations as a function of both collision energy and pseudorapidity region has already been successfully tested in [4].

Let us now plot the parameter variations as a function of the rapidity interval between the incoming proton and the produced secondary hadron in the moving proton rest frame according to a simple formula:

$$
\eta^{\prime}=|\eta|-\log \left(\sqrt{s} / 2 m_{p}\right), \quad \eta^{\prime \prime}=|\eta|+\log \left(\sqrt{s} / 2 m_{p}\right),
$$

where $m_{p}$ is the mass of the incoming proton. The results of this procedure are shown in figure 2. Surprisingly, all the points came to a single line in this interpretation. To understand the origin of this universality one might use Monte $\operatorname{Carlo}(\mathrm{MC})$ generators: hard processes at large $p_{T}$ are known to be described by MC generators pretty well, thus it is expected to get the value of the Nparameter from the fits of the MC-generated spectra rather close to the real data, but with a higher accuracy and in a wider collision energy range. To check this universality, we have produced the Monte Carlo samples for proton-proton collisions at different energies for inelastic(INEL) events with the PYTHIA 8.2 generator. Indeed, the values of the parameter $\mathrm{N}$ extracted from the fits to the MC-generated spectra are nicely placed at the same line. Thus, a universal parameter describing the shape of the transverse momentum spectra in pp-collisions has been found.

Remarkably, similarly to $N$, the $T$ and $T_{e}$ also show dependences as a function of both the collisions energy $\sqrt{s}$ and the measured pseudorapidity interval $\eta$. The variations of the $T$ and $T_{e}$ parameters were studied in [4]. The dependences are shown on figure 2 as well.

\section{Prediction for further measurements}

Using the parameter dependences [5] extracted from figure 2, one can calculate double dif- 

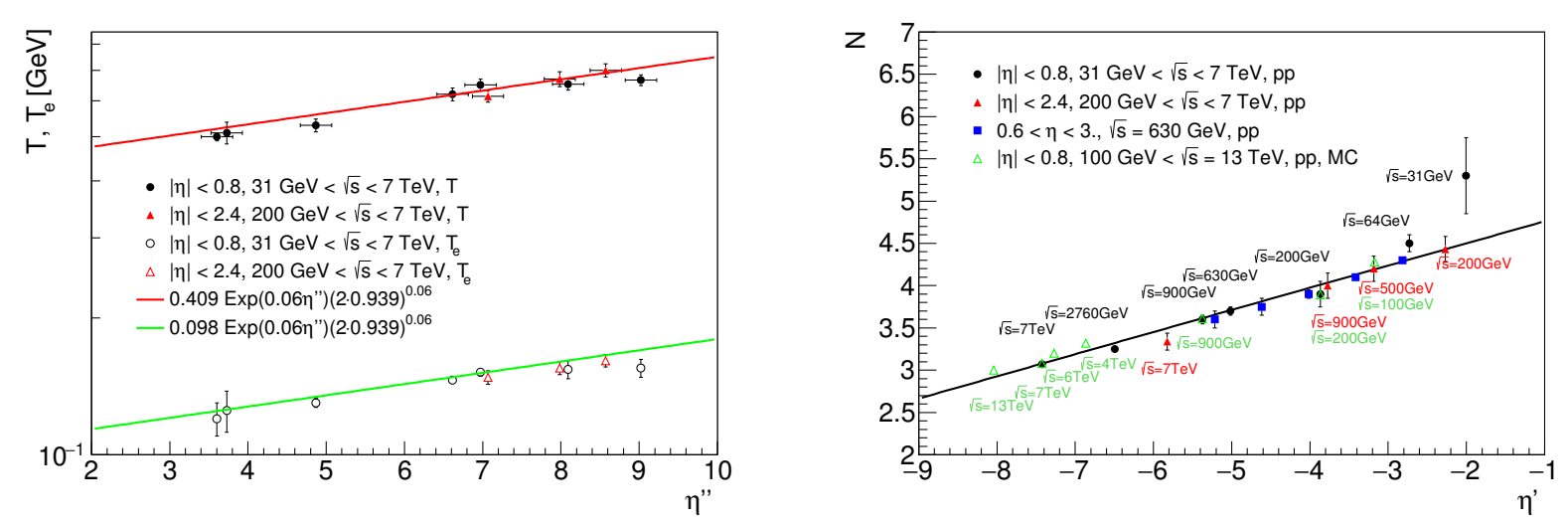

Figure 2: The dependence of the parameters on the pseudorapidity of the secondary hadron in the moving opposite side proton rest frame.

ferential cross sections $d^{2} \sigma /\left(d \eta d p_{T}^{2}\right)$ of charged particle production in high energy collisions at different energies for NSD events. These predictions are shown in figure 3 for $|\eta|<0.8$ and $|\eta|<2.4$ pseudorapidity intervals together with the experimental data measured by CMS and ALICE. A good agreement of the prediction with the data can be observed. Thus, these results give us a powerful tool for predicting the spectral shapes in NSD events.

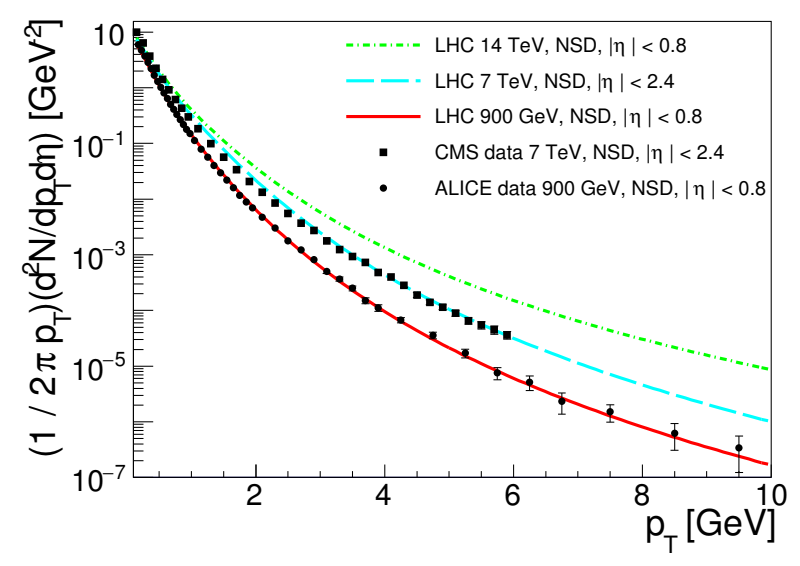

Figure 3: Predictions of the yield of charged particles $\left(1 / 2 \pi p_{T}\right) d^{2} N /\left(d \eta d p_{T}\right)$ in high energy collisions in NSD events together with data points from the ALICE [7] and CMS [6] experiments.

\section{Possible Interpretation}

The universal thermal character of hadron transverse momentum spectra and abundances in all high energy processes can hardly be a coincidence and begs for a theoretical explanation. One attempt to understand it is based on the hypothesis that confinement is associated with an event horizon for colored particles. The quantum effects then produce the thermal spectra of hadrons, similarly to the Hawking evaporation of black holes or Unruh radiation. The color string stretching between the colored fragments in a high energy collision contains the longitudinal chromoelectric 
field. This field deccelerates the colored fragments producing a Rindler event horizon. Quantum fluctuations in the vicinity of the event horizon then result in the thermal production.

The effective temperature of the hadron spectrum in this picture is proportional to decceleration that is driven by the confining chromoelectric field. The strength of the chromoelectric field at low collision energies is determined by the string tension. At high energies, the quantum evolution effects come into play, increasing the number of gluons in the wave functions of the colliding hadrons; therefore the chromoelectric field becomes stronger.

An economic and theoretically consistent way to describe this phenomenon is offered by the parton saturation, or color glass condensate, picture. In this approach the density of partons in the transverse plane inside hadrons, and thus the strength of the color field after the hadron collision, is parameterized by the saturation momentum $Q_{s}(s, \eta)$ that depends on the c.m.s. collision energy squared $s$ and (pseudo-)rapidity $\eta$. The decceleration $a$ then appears proportional to the value of the saturation momentum, $a \sim Q_{s}$. The temperature of the radiation from the resulting Rindler event horizon is thus given by

$$
T_{t h} \propto \frac{Q_{s}}{2 \pi} .
$$

The saturation momentum itself can be parametrized in the following way

$$
Q_{s}^{2}(s ; \pm \eta)=Q_{s}^{2}\left(s_{0} ; \eta=0\right)\left(\frac{s}{s_{0}}\right)^{\lambda / 2} \exp ( \pm \lambda \eta)
$$

where $\lambda \simeq 0.2 \div 0.3$ is the intercept. In the saturation scenario, $Q_{s}$ is the only dimensionful parameter, so the transverse momentum spectra $F\left(p_{T}\right)$ have to scale as a function of $p_{T} / Q_{s}$ :

$$
F\left(p_{T}\right)=F\left(p_{T} / Q_{s}\right)
$$

Let us now check whether the relation between $T_{t h}$ and $T$ is indeed linear. Since the variations of the temperature-like parameters $T$ and $T_{t h}$ as a function of pseudorapidity are expected from (4.2), it is desirable to exclude their influence when studying the dependences of these parameters on the c.m.s. energy in a collision. This is possible if one combines only the data in more or less the same pseudorapidity intervals. Hence we look first at ISR, PHENIX, ALICE and UA1 data in the most central $(|\eta|<0.8)$ pseudo-rapidity region.

Figure 4 shows variations of $T$ and $T_{t h}$ as a function of c.m.s. energy in a collision. One can describe the energy dependence by the power-law fits (4.2) shown in figure 4:

$$
T=409 \cdot(\sqrt{s})^{0.06} \mathrm{MeV}, T_{t h}=98 \cdot(\sqrt{s})^{0.06} \mathrm{MeV} .
$$

Remarkably, from (4.4) one can again notice the linear relation between $T$ and $T_{t h}$.

To study the variations of $T$ and $T_{\text {th }}$ parameters as a function of pseudorapidity one can use the data published by the UA1 experiment. Figure 4 shows these variations together with the lines standing for the exponential behavior predicted from eq. (4.2) with $\lambda=0.12$ as obtained from the fits (4.4).In addition, figure 4 shows UA1, BRAHMS and CMS data measured under different experimental conditions. In these measurements the pseudo-rapidity interval was much wider. Therefore, one can compare the parameter values obtained from the fits of these data (open points in figure 4) to the values calculated according to (4.2) with $\lambda=0.12, T^{0}$ and $T_{t h}^{0}$ taken 

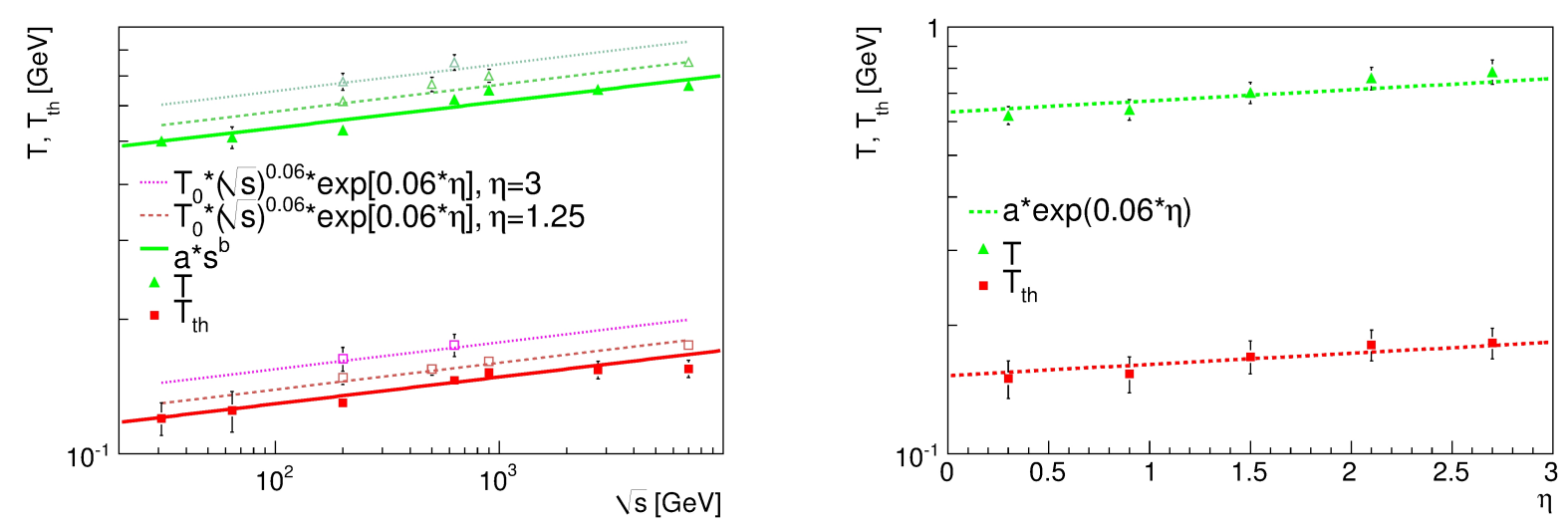

Figure 4: Variations of the $T, T_{t h}$ parameters obtained from the fits to the experimental data as a function of $\sqrt{s}$ (left) and $\eta$ (right). In the left plot full points represent the data measured in the central rapidity region, while open points stand for measurements performed in the forward region, lines show power-law fits of these variations: solid in the central region and dashed in the forward region.

from (4.4) and $\eta$ taken as the mean value of the measured pseudorapidity interval. Rather good agreement between these predictions and the experimental data can be observed from figure 4 further supporting the proposed behavior.

We hope that our analysis sheds some light on the origin of the thermal component in hadron production. The established proportionality of the parameters describing the "thermal" and "hard" components of the transverse momentum spectra supports the theoretical picture in which the soft hadron production is a consequence of the quantum evaporation from the event horizon formed by deceleration in longitudinal color fields. The absence of the thermal component in diffractive interactions lend further support to our interpretation. It will be worthwhile to extend this analysis to other high energy processes. Future precise measurements at LHC are needed to further study the proposed picture for hadron production.

\section{Conclusion}

In conclusion, transverse momentum spectra in $p p$-collisions have been considered using a two component model. Variations of the parameters obtained from the fit have been studied as a function of pseudorapidity $\eta$ and c.m.s. energy $\sqrt{s}$ in the collision. A universal parameter describing a shape of the spectra in pp-collisions was found to be a preudorapidity of a secondary hadron in the moving proton rest frame. Finally, the observed dependences, together with previous investigations allowed to make predictions on double differential spectra $d^{2} \sigma /\left(d p_{T}^{2} d \eta\right)$ at higher energies, successfully tested on the available experimental data.

\section{References}

[1] A. A. Bylinkin and A. A. Rostovtsev, Phys. Atom. Nucl. 75 (2012) 999.

[2] A. A. Bylinkin, M. G. Ryskin, Phys. Rev. D 90 (2014) 1, 017501

[3] A. A. Bylinkin and A. A. Rostovtsev, Nucl. Phys. B 888 (2014) 65 
[4] A. A. Bylinkin, D. E. Kharzeev and A. A. Rostovtsev, Int. J. Mod. Phys. E 23 (2014) 12, 1450083

[5] A. Bylinkin, N. S. Chernyavskaya and A. A. Rostovtsev, Eur. Phys. J. C 75 (2015) 4, 166

[6] V. Khachatryan et al. [CMS Collaboration], Phys. Rev. Lett. 105 (2010) 022002

[7] K. Aamodt et al. [ALICE Collaboration], Phys. Lett. B 693 (2010) 53-68 